\title{
How being synanthropic affects the gut bacteriome and mycobiome: comparison of two mouse species with contrasting ecologies
}

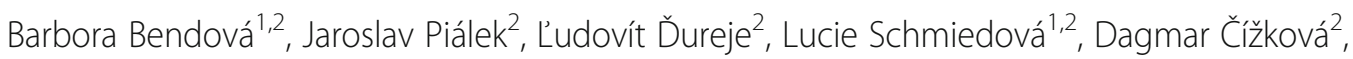 \\ Jean-Francois Martin ${ }^{3}$ and Jakub Kreisinger ${ }^{1 *}$ (D)
}

\begin{abstract}
Background: The vertebrate gastrointestinal tract is colonised by microbiota that have a major effect on the host's health, physiology and phenotype. Once introduced into captivity, however, the gut microbial composition of freeliving individuals can change dramatically. At present, little is known about gut microbial changes associated with adaptation to a synanthropic lifestyle in commensal species, compared with their non-commensal counterparts. Here, we compare the taxonomic composition and diversity of bacterial and fungal communities across three gut sections in synanthropic house mouse (Mus musculus) and a closely related non-synanthropic mound-building mouse (Mus spicilegus).

Results: Using Illumina sequencing of bacterial 165 rRNA amplicons, we found higher bacterial diversity in M. spicilegus and detected 11 bacterial operational taxonomic units with significantly different proportions. Notably, abundance of Oscillospira, which is typically higher in lean or outdoor pasturing animals, was more abundant in non-commensal M. spicilegus. ITS2-based barcoding revealed low diversity and high uniformity of gut fungi in both species, with the genus Kazachstania clearly dominant.

Conclusions: Though differences in gut bacteria observed in the two species can be associated with their close association with humans, changes due to a move from commensalism to captivity would appear to have caused larger shifts in microbiota.
\end{abstract}

Keywords: Microbiome, Metabarcoding, Steppe mouse, Muridae, Symbiosis, Evolution

\section{Background}

Various animal species benefit from a commensal association with humans, which offers advantages in terms of extended and more predictable food supplies, lower predation pressure and an ability to spread outside of their original distribution range [1-3]. At the same time,

\footnotetext{
* Correspondence: jakubkreisinger@seznam.cz

'Department of Zoology, Faculty of Science, Charles University, Prague, Czech Republic

Full list of author information is available at the end of the article
}

however, commensals may suffer from higher humaninduced mortality [4] and a higher infection risk from pathogens specific to humans or other humanassociated animals [5]. Moreover, abiotic and biotic factors associated with human-altered and ancestralhabitat environments of human-associated symbionts differ, inducing corresponding changes in selective pressures acting on commensal phenotypes [6]. Consequently, individuals from symbiotic populations may differ morphologically, physiologically and behaviourally

(c) The Author(s). 2020 Open Access This article is licensed under a Creative Commons Attribution 4.0 International License, which permits use, sharing, adaptation, distribution and reproduction in any medium or format, as long as you give appropriate credit to the original author(s) and the source, provide a link to the Creative Commons licence, and indicate if changes were made. The images or other third party material in this article are included in the article's Creative Commons licence, unless indicated otherwise in a credit line to the material. If material is not included in the article's Creative Commons licence and your intended use is not permitted by statutory regulation or exceeds the permitted use, you will need to obtain permission directly from the copyright holder. To view a copy of this licence, visit http://creativecommons.org/licenses/by/4.0/ The Creative Commons Public Domain Dedication waiver (http://creativecommons.org/publicdomain/zero/1.0/) applies to the data made available in this article, unless otherwise stated in a credit line to the data. 
from their wild ancestors (e.g. see [7-10]). Despite intensive research in this field, several aspects of phenotypic change due to a commensal lifestyle remain unexplored. In particular, little is known about the effect of a commensal lifestyle on the composition and function of microbial communities harboured within commensal bodies [11] and putative phenotype changes that may be induced by variation in microbial populations.

Animal-associated microbial composition has an important influence on both host health and physiology [12-14], including digestive capacity [15] and interactions with the host's central nervous [16] and immune systems $[17-20]$ and, as such, can be considered a component of the animal's phenotype. At the same time, animal-associated microbiota exhibit considerable plasticity due to changing physiological states and environmental conditions, including diet variation, stress or parasite infection [16, 21-23]. Consequently, there is a high expectation that remodulation of a host's microbiota due to a commensal lifestyle may parallel widely observed changes in microbial populations after translocation of free-living individuals into captivity [24-27].

Murine rodents represent a suitable model group for exploring the effect of commensalism on associated microbiota as the switch between a commensal vs. noncommensal lifestyle has taken place repeatedly during the course of their evolution $[1,28]$. To date, however, most research on murine gut microbiota has focused on captive murine species [12, 15, 21, 29-32], commensal populations of murine rodents [33-35] or noncommensal murine species that are phylogenetically distant to commensal taxa [11,36]. Consequently, there is little comparative data available aimed directly at the assessment of commensalism on microbial structure. This lack of knowledge is even greater as regards eukaryotic microbiota, with the major component in noncommensal animals, the fungal mycobiome, having only been studied exceptionally [37], and never characterised in wild rodents.

To gain an insight into microbial changes due to commensalism in murine rodents, we studied the gut microbiota of two closely related mouse species, the mound-building mouse (Mus spicilegus; hereafter MS) and the house mouse (Mus musculus; hereafter MM). Together with two other mouse species, MM and MS form a monophyletic group with a genetic distance between them of less than 1\% [38]. MS are found in the steppes or in agricultural landscapes and use large mounds built from harvested plants as communal shelters and reproduction sites; importantly, the MS lifecycle is independent of human buildings [39]. Unlike the noncommensal MS, MM benefit from a tight commensal association with humans established ca. 8000 years ago that has enabled its cosmopolitan spread [1].
Consequently, the MM lifecycle is dependent on human infrastructure over most of its current distributional range, including our sample sites.

In our study, we use culture-independent gut microbiota profiling based on high-throughput amplicone sequencing, focusing specifically on the gut bacteriome (hereafter GB), which was characterised using 16S rRNA profiling. Moreover, for the first time, our research describes the gut mycobiome (hereafter GM) structure of a free-living mouse population using ITS2 sequencing. As gut microbial variation in different gut sections from the same individual may exceed interindividual variation of microbial communities sampled within single gut compartments [35], we assessed whether there are any species-specific patterns in gut microbial variation across three gut sections (colon, caecum and ileum).

\section{Results}

\section{Gut bacteriome}

GB profiles of the two mouse species were dominated by bacteria of the phyla Firmicutes (40\% of all reads), Proteobacteria (25.3\%), Bacteroidetes (20\%), Tenericutes (7.4\%) and Deferribacteres (6.1\%, Fig. 1). Another six phyla were detected in our dataset (Spirochaetes, TM7, Actinobacteria, Cyanobacteria, Fusobacteria and Acidobacteria) at low abundances $(<1 \%$ reads). At both the phylum and class level, GB content was comparable between MM and MS; however, the dominant bacterial phyla and classes tended to vary between gut sections (Fig. 1, Table S1). In particular, Bacilli (phylum Firmicutes, represented by the genus Lactobacillus), Mollicutes (phylum Tenericures, represented by the genus Mycoplasma) and Gammaproteobacteria (Proteobacteria, represented by the genus Aggregatibacter) were more common in the ileum than the colon or caecum (Fig. 1, Table S1). On the other hand, Bacteroidia (phylum Bacteroidetes, represented by the genera Bacteroides and Odoribacter, unassigned S24-7 and Rikenellaceae), Clostridia (phylum Firmicutes, represented by unspecified Lachnospiraceae and the genera Oscillospira and Ruminococcus) and Epsilonproteobacteria (phylum Proteobacteria, represented mainly by the genus Helicobacter) were consistently dominant in the colon and caecum.

GB alpha diversity varied between gut sections (LME: $\Delta D F=2, x^{2}=10.62, p=0.005$, Table 1, Fig. 2). Specifically, GB was less diverse in the ileum compared to the colon or caecum (Tukey post-hoc comparison: $p<0.05$ in both cases), whereas the diversity of colon and caecal GB was comparable (Tukey post-hoc comparison: $p=$ 0.8395). After the statistical control for this source of variation, $\mathrm{GB}$ was more diverse in non-commensal MS compared to commensal MM (LME: $\Delta \mathrm{DF}=1, \mathrm{x}^{2}=6.17$, $p=0.013)$. At the same time, however, variation in 


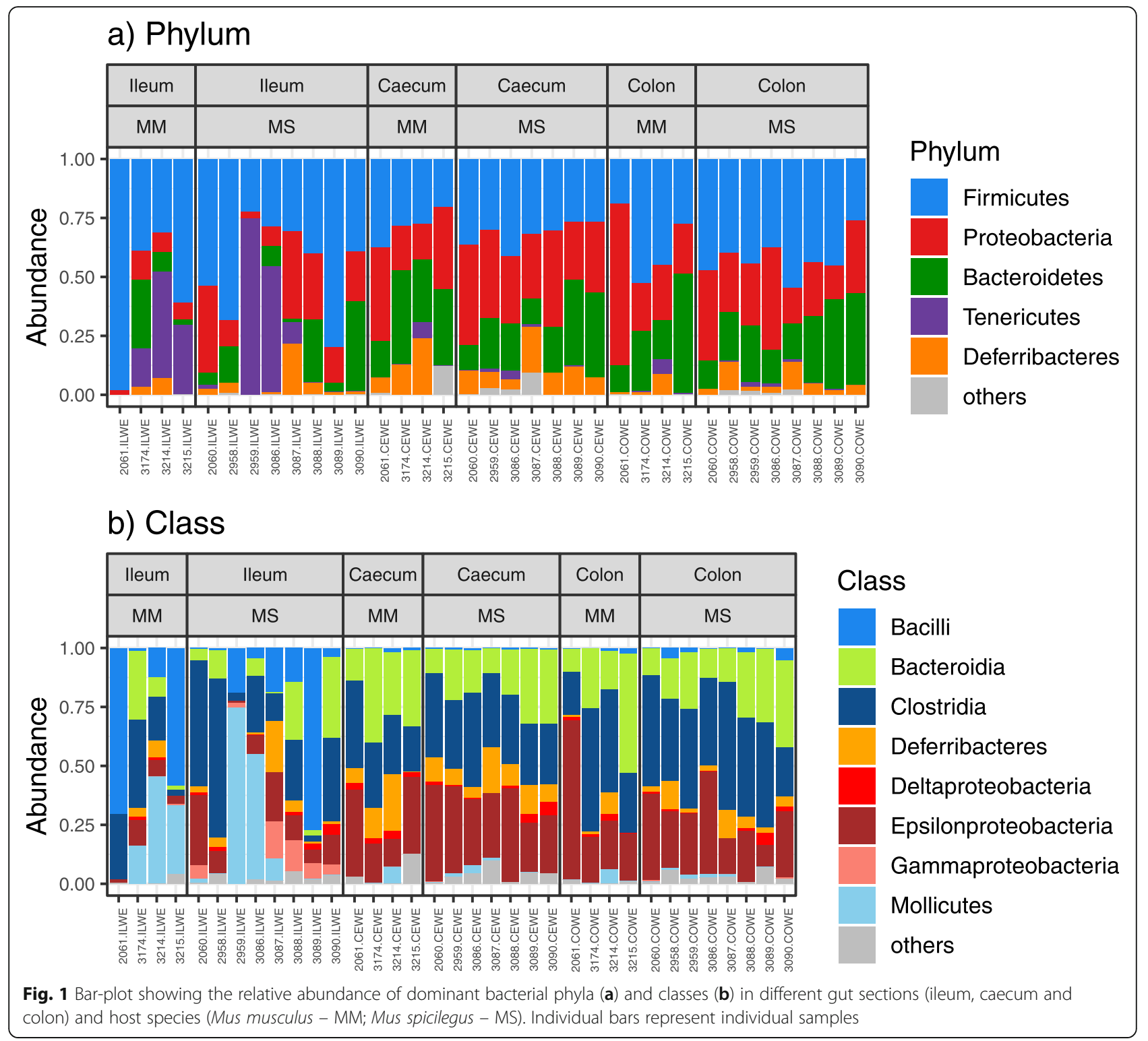

microbial diversity between gut sections showed comparable pattern in the two species, as indicated by nonsignificant species identity vs. gut section interaction (LME: $\Delta \mathrm{DF}=2, \mathrm{X}^{2}=3.64, p=0.162$ ). After statistical control for variation between species and gut sections, neither sex (LME: $\triangle \mathrm{DF}=1, \mathrm{X}^{2}=0.06, p=0.811$ ) nor sample location (LME: $\Delta \mathrm{DF}=1, \mathrm{X}^{2}=0.73, p=0.393$ ) had any effect on GB diversity.

According to both OTU prevalence-based (i.e. Jaccard) and relative abundance-based (i.e. Bray-Curtis)

Table 1 Minimum adequate model describing the effect of predictors (gut section and species identity) on gut bacteriome alpha diversity variation (Shannon index) in Mus spicilegus (MS) and Mus musculus (MM). The MS ileal gut bacteriome was used as the reference level (i.e. Intercept) in minimum adequate model parametrization

\begin{tabular}{|c|c|c|c|c|c|}
\hline & Estimate & Std. Error & Df & $\mathrm{t}$ value & $P$ value \\
\hline (Intercept) & 2.389 & 0.260 & 31 & 9.187 & $<0.001$ \\
\hline Gut section (ileum vs. caecum) & 0.728 & 0.290 & 31 & 2.508 & 0.018 \\
\hline Gut section (ileum vs. colon) & 0.857 & 0.284 & 31 & 3.017 & 0.005 \\
\hline Species (MM vs. MS) & 0.638 & 0.248 & 31 & 2.575 & 0.015 \\
\hline
\end{tabular}



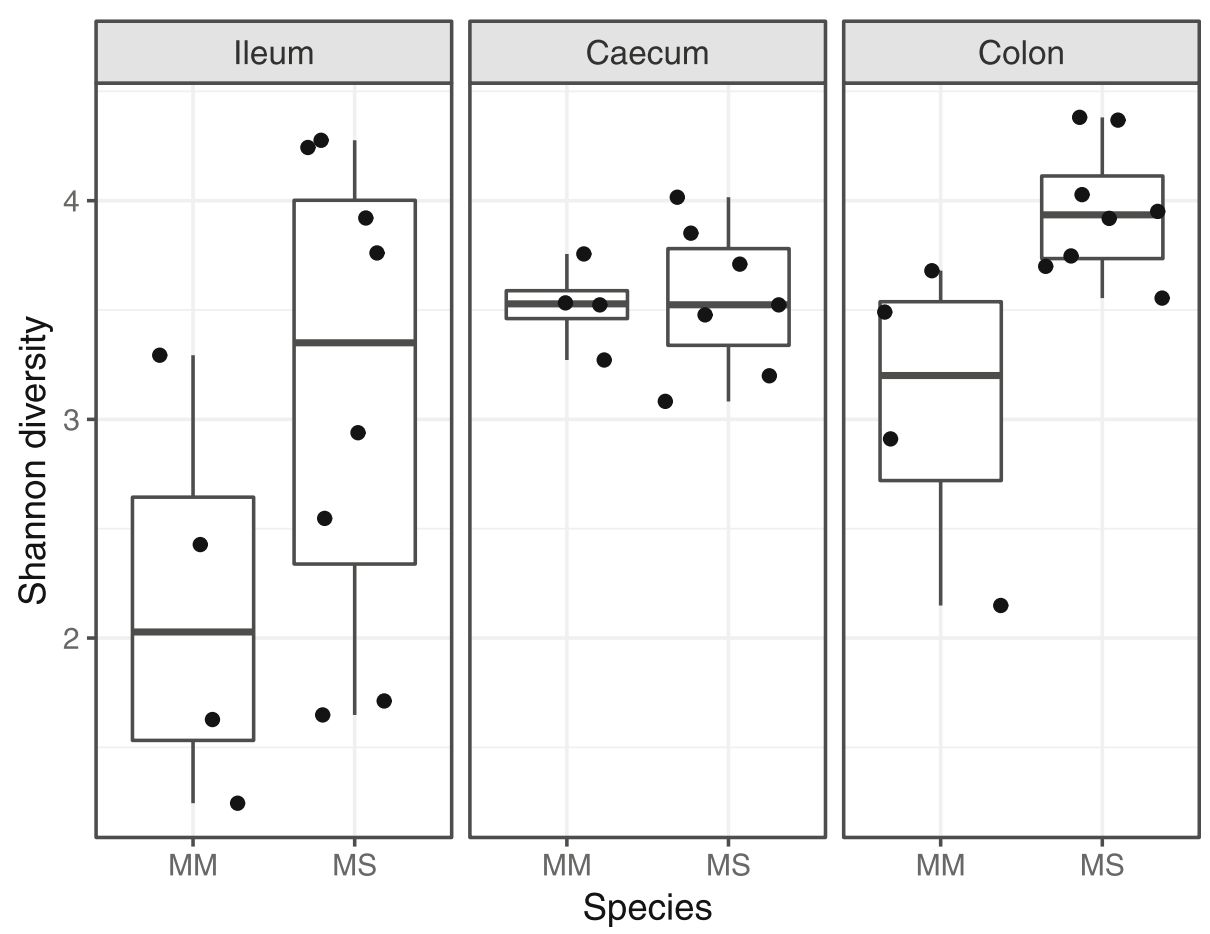

Fig. 2 Box-plot showing variation in gut bacteriome Shannon diversity between gut sections (ileum, caecum and colon) and host species (Mus musculus - MM; Mus spicilegus - MS)

dissimilarities, MM vs. MS exhibited divergent GB content associated with the first PCoA axis. In addition, the second PCoA axis tended to separate individuals from different locations. Surprisingly, however, PCoA suggested only slight GB divergence between gut sections (Fig. 3). PERMANOVA analysis indicated that the effect of species and sample location was highly significant for both dissimilarity types, whereas differences between gut sections were only supported by PERMANOVA for relative abundance-based dissimilarity. The effect of sex, despite being marginally significant, explained only $<5 \%$ of GB variation (Table 2).

Using negative binomial GLMMs, we detected 11 OTUs (represented by $11 \%$ of all reads in our dataset) whose abundances varied between MM and MS. Remarkably, four Oscillospira, two OTUs from the family Clostidiaceae and one Odoribacter OTU were more abundant in non-commensal MS than commensal MM. The two species also differed in relative abundance of two Helicobacter OTUs, one being more abundant in MM and the other in MS (Fig. 4). According to phylogenetic placement analysis, these OTUs represent phylogenetically distant Helicobacter species, where Campylobacter jejuni (GenBank accession: EU127548.1) is considered as a root (Fig. S1). Furthermore, OTUlevel analysis identified 55 OTUs (representing 71\% of all reads) whose abundances varied between gut sections (Fig. 5).

\section{Gut mycobiome}

Unlike the GB, GM profiles were highly homogenous across all murine samples, with fungi of the genus Kazachstania consistently dominant (representing 97\% of all reads and 28 OTUs). According to species-level assignment, most samples comprised $K$. pintolopesii, with the GM of a single MM individual being dominated by $K$. heterogenica. Other fungal taxa were represented by a low proportion of reads (Fig. 6). Alongside the murine samples, we also undertook GM profiling (using the same methodology) of ten faecal microbiota samples from a passerine bird (barn swallow [Hirundo rustica], see [40] for details). The GM composition of these samples was much more diverse than the murine GM and covered a broader range of phylogenetically distant fungal taxa. Moreover, Kazachstania was almost absent in these non-murine samples (Fig. S2). As such, we conclude that the high homogeneity and low diversity of the murine GM profiles did not arise as an artefact of wet laboratory procedures.

\section{Discussion}

Changes in gut microbiota due to association with humans have been described for a range of vertebrate species [11, 24-27, 34, 41, 42] living in wild vs. in captivity, i.e. zoological gardens [27, 42], domesticated animals $[26,41]$ and laboratory animals $[34,43]$. Overlaying wild vs. captivity microbiome changes for different species 


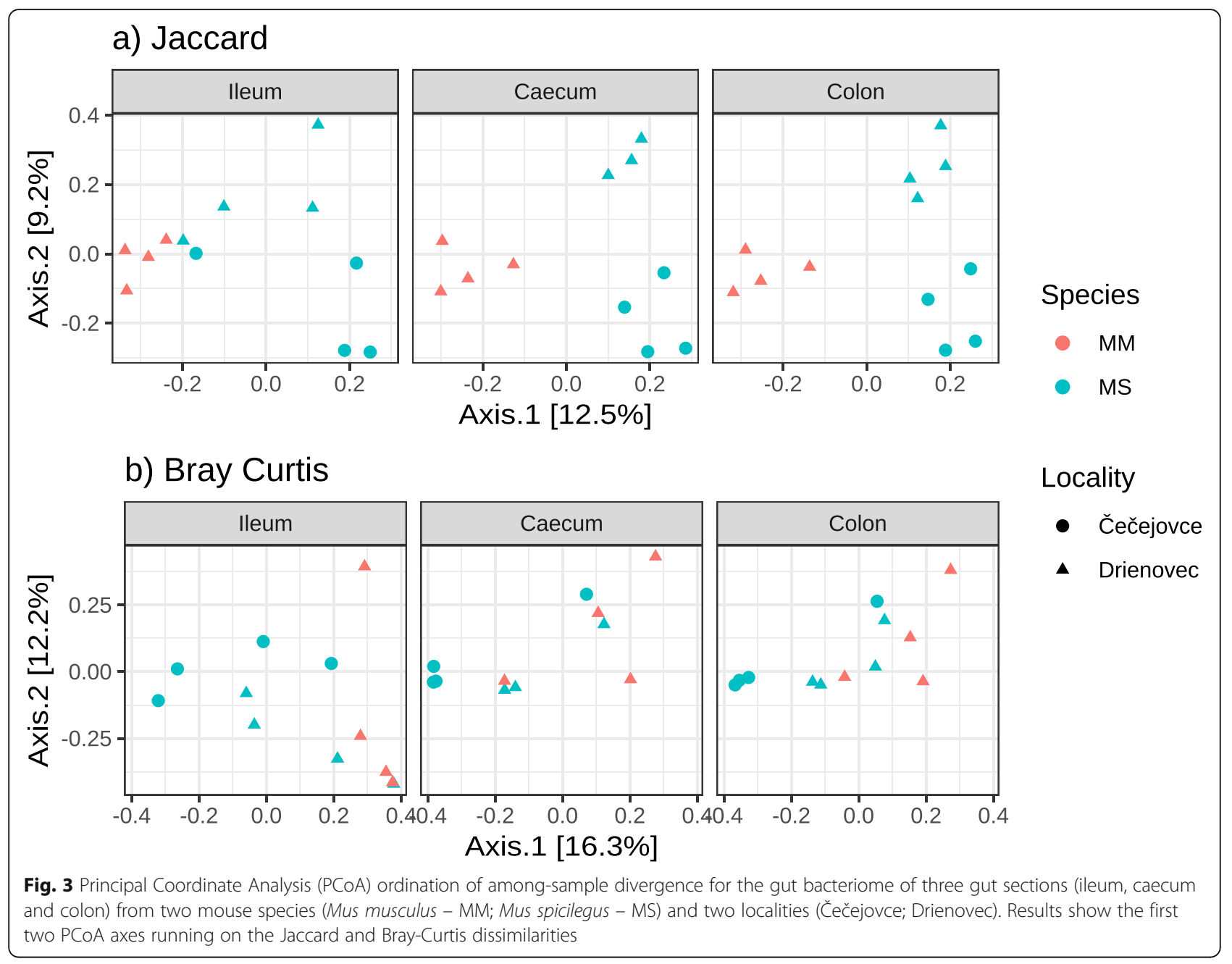

Table 2 Results of PERMANOVA analysis testing for the effect of sex, sample location, gut section and species identity on variation in gut bacteriome composition. The Table presents results for absence vs. presence (Jaccard) and relative abundance (Bray-Curtis) dissimilarities not accounting for operational taxonomic unit phylogeny

\begin{tabular}{lllllll}
\hline Dissimilarity & Variable & Df & Sum Of Sqs. & F value & P value & $R^{2}$ \\
\hline Jaccard & sex & 1 & 0.621 & 1.891 & 0.004 & 0.047 \\
& locality & 1 & 1.090 & 3.320 & $<0.001$ & 0.083 \\
& mouse species & 1 & 1.306 & 3.979 & $<0.001$ & 0.099 \\
& gut section & 2 & 0.664 & 1.012 & 0.436 & 0.050 \\
& residual & 29 & 9.520 & & & 0.721 \\
Bray-Curtis & sex & 1 & 0.603 & 2.077 & 0.007 & 0.049 \\
& locality & 1 & 1.117 & 3.851 & $<0.001$ & 0.092 \\
& mouse species & 1 & 1.052 & 3.626 & $<0.001$ & 0.086 \\
& gut section & 2 & 0.993 & 1.711 & 0.008 & 0.082 \\
& residual & 29 & 8.414 & & & 0.691 \\
\hline
\end{tabular}

potentially allows for the identification of general patterns of microbiome change due to association with humans. On the other hand, the opportunity to establish altered (co)adaptations between microbiota and their hosts in the context of human-altered living environments and lifestyle of the host is limited in captivity. A change in the microbiota of the first generation of animals bred in captivity, for example, may simply represent an unstable, transient state that does not yet show shifts typical for human associated lifestyles. Here, we contrast the gut microbiome of two closely-related mouse species adapted to ecological niches differing greatly in tightness of association with humans (the commensal house mouse [MM] and the non-commensal mound-building mouse [MS]), to get insight on how can commensal association with humans affect bacterial and fungal communities residing in the gut.

In our study, MS displayed a higher gut bacteriome diversity than MM. This may parallel the commonly observed decrease in microbial diversity after introduction of wild animals to captivity [24, 25, 42, 44], ascribed to 


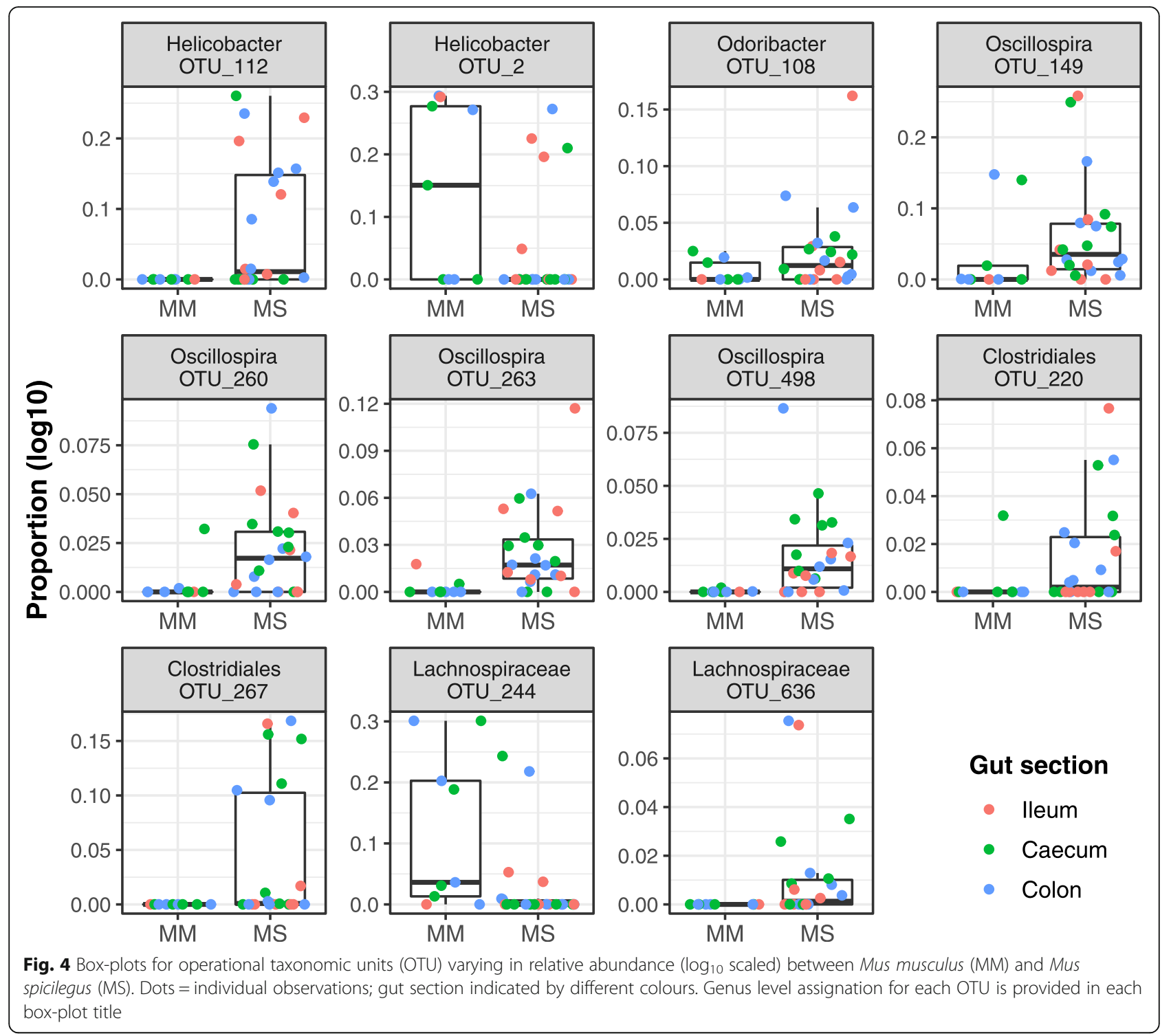

reduced variation either in environmental factors affecting GB richness (such as diet composition) or in environmental bacteria colonising gut. While GB diversity between the two mouse species showed significant differences, GB diversity variation between different gut sections was even more dramatic, with ileal GB being less diverse than that from the colon and caecum. These within-gut diversity changes are consistent with most previous studies on different mammalian taxa, including rodents $[32,35,36]$, and can be explained by spatial gradients in immune function, acidity level, nutrient concentrations and various host-derived secretions within the gut $[30,45,46]$.

In addition to changes in GB diversity, we also observed differences in GB composition between the two species. Subsequent differential-abundance analysis identified 11 OTUs (represented by $11 \%$ of high-quality reads) whose proportions varied between the two species, with the greatest difference being the increase in abundance of four Oscillospira OTUs in MS. Interestingly, in parallel to our findings, a previous study on cattle showed that individuals relying on outdoor pasture hosted more abundant Oscillospira populations than indoor-bred individuals [47]. According to a few independent studies on human subjects, an increase in Oscillospira abundance was associated with reduced body weight [48]. Moreover, a comparative study on several vertebrate species exposed to a fasting regime revealed consistent enrichment of their GB by Oscillospira [44]. Together, these observations suggest that variation in the abundance of Oscillospira OTUs between MS and MM may reflect differences in nutritional conditions between the commensal and non-commensal niches. Along with Oscillospira, two OTUs from the family 


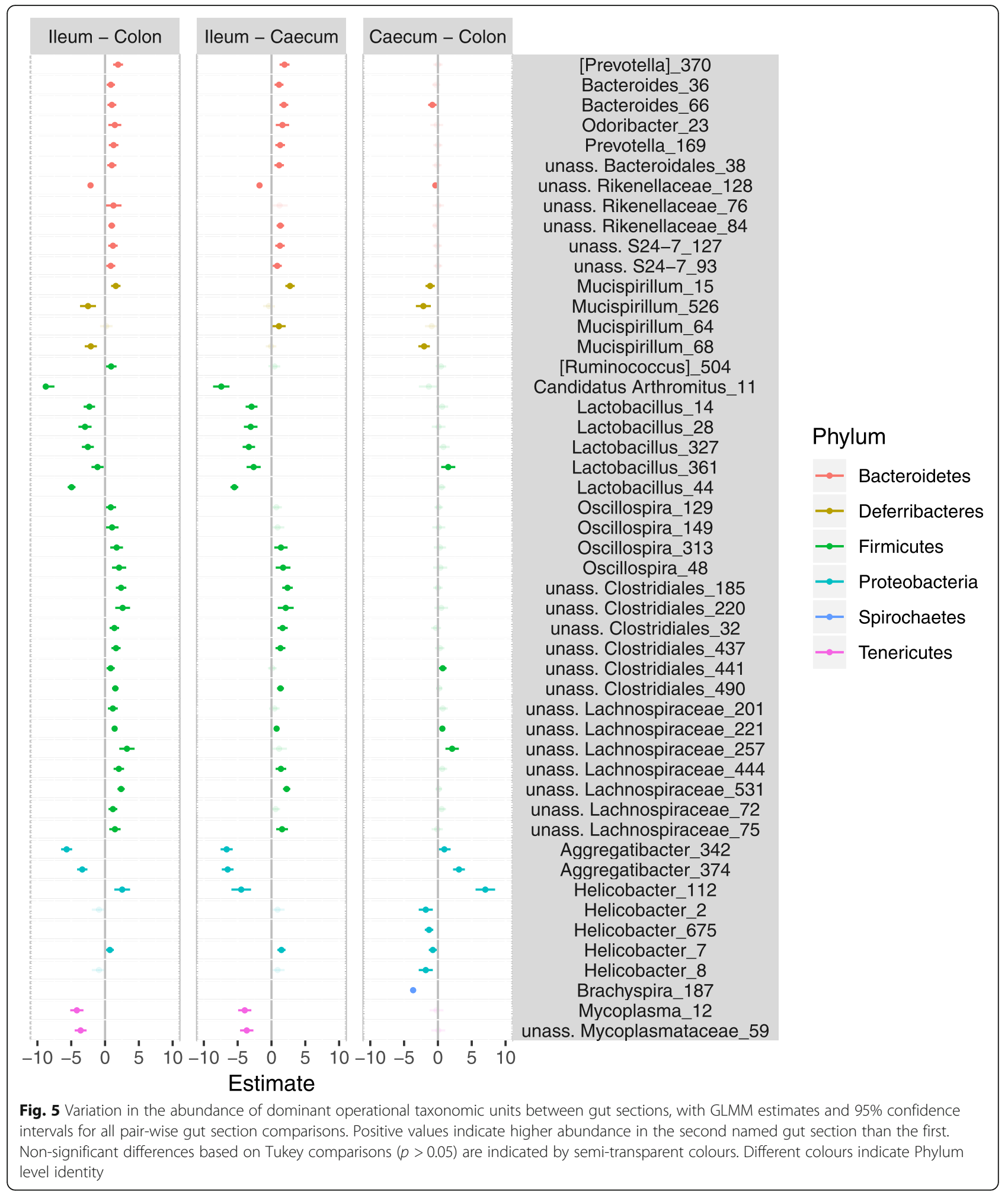

Clostridiaceae and one Odoribacter OTU were more abundant in non-commensal MS than commensal MM. All these taxa (including Oscillospira) are anaerobes, capable of fermenting complex plant polysaccharides and producing bioactive short-chained fatty acids
(SCFA) with anti-inflammatory capacity $[49,50]$. As deficit of SCFA is associated with increased emergence of metabolic disorders and impaired cognitive and immune functions [51], an experimental study linking differences in the abundance of specific SCFA-producing bacteria to 


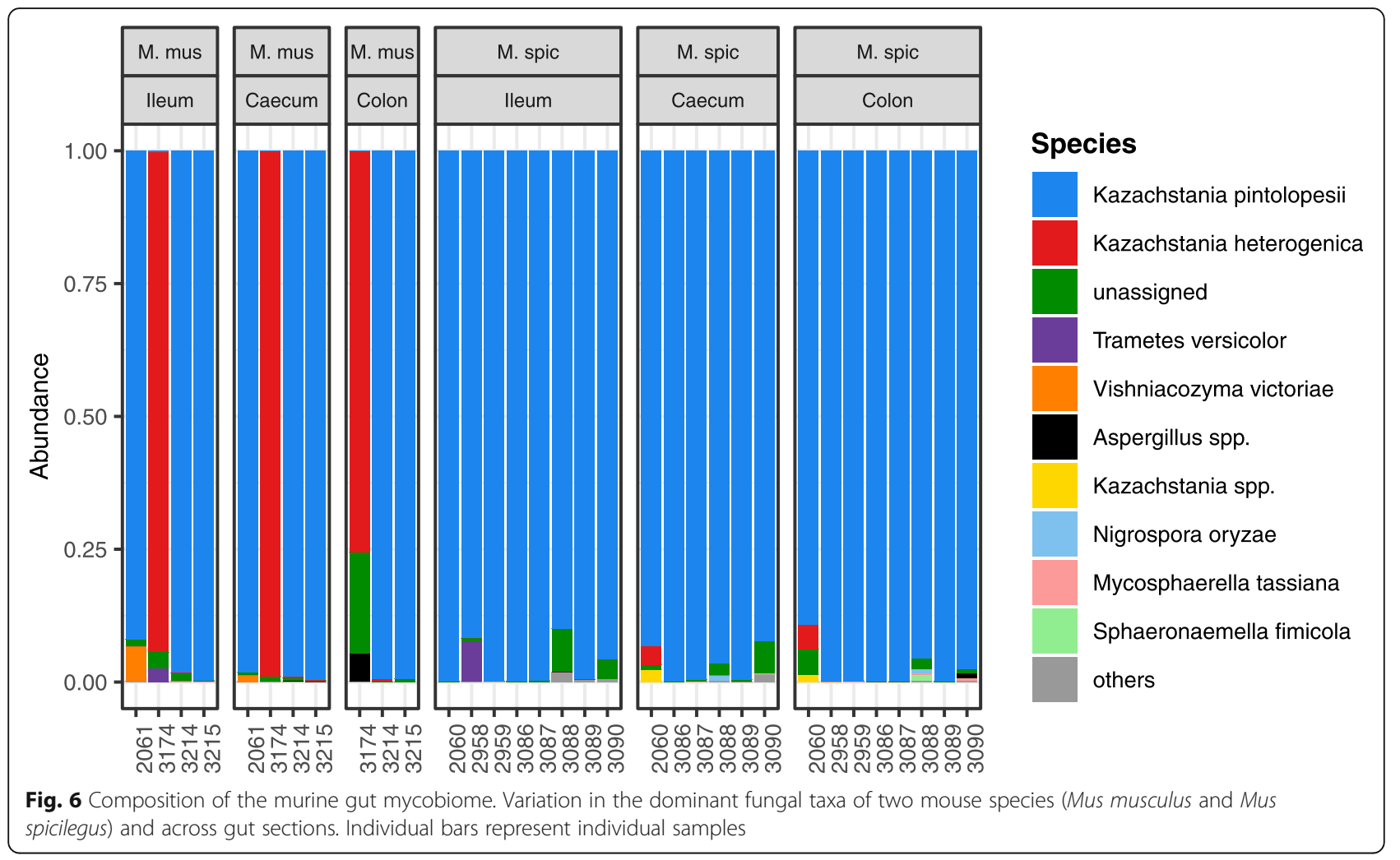

potential differences in immune and physiological function between MM and MS would be of great interest., MM and MS gut bacteriome also differed in abundance of two Helicobacter and two Lachnospiraceae OTUs. Helicobacter, a common inhabitant of murine GB [36, 52], can induce pathological states under certain circumstances [29, 53]. Some Helicobacter species are transferred across generations by social contact between community members and, consequently, exhibit phylogeographic codivergence with their hosts $[54,55]$. This does not appear to be the case regarding the differences in abundance of Helicobacter OTUs in MM and MS, however, as they represent phylogenetically distant lineages and group with Helicobacter clades of low host specificity. Particularly, Helicobacter OTUs abundant in MS exhibited relatedness to $H$. canicola and $H$. bilis, whereas those abundant in MM clustered with $H$. apodemus.

In this study, considerable differences in GB composition were observed between gut sections, irrespective of host species identity, with the abundance of 55 OTUs (representing $71 \%$ of all reads) varying between gut sections. Variation in GB taxonomic content between gut sections was generally congruent with most previously published data on mammalian species [35, 36]. In particular, the distal parts of the gut (i.e. the colon and caecum) were enriched with OTUs of the phylum Bacteroidetes (taxa Bacteroides, Prevotella, S24-7, Rikenellaceae), class Clostridia (genera Ruminococcus, Oscillospira and unassigned Lachnospiraceae) and the genus Helicobacter (from phylum Proteobacteria). On the other hand, the ileal GB was characterised by an increased abundance of Candidatus Arthromitus, Lactobacillus (both of the phylum Firmicutes), Mycoplasma (phylum Tenericutes), Aggregatibacter (phylum Proteobacteria) and Mucispirillum (phylum Defferibacteres). GB composition also varied between sample locations, implying that the individuals sampled were exposed to different environmental bacterial pools, despite being only $10 \mathrm{~km}$ apart, which is consistent with previous studies on wild MM microbiota [33, 43]. Finally, males and females exhibited only slight variation in $\mathrm{GB}$, accounting for $\sim 4 \%$ of total variation in GB composition.

Surprisingly, both MM and MS exhibited highly uniform gut mycobiome structure, with the genus Kazachstania (including two species, $K$. pintolopesii and $K$. heterogenica) as the dominant component, representing ca. $75-100 \%$ of high-quality reads. Kazachstania, a species complex of Ascomycetous yeasts, has been isolated from a number of captive species, including $K$. heterogenica and $K$. pintolopesii from rodents, $K$. slooffiae from pigs and horses and K. bovina from cows and birds [56]. Although little is known about its functions, recent experimental studies have shown the importance of Kazachstania for the development of a healthy porcine microbiome, where Kazachstania populations support growth of SCFA-producing bacteria [57, 58]. On the 
other hand, presence of Kazachstania in Mongolian gerbils (Meriones unguiculatus) exacerbated pathologic effects after experimental infection with Helicobacter suis [59]. Similarly, mixed infection of Kazachstania and Escherichia coli was reported as a causal agent of a fatal disease in captive bred primates [60]. Interestingly, the GM of free-living mice from our populations differed from GM profiles previously reported for captive MM, with captive individuals exhibiting much higher diversity and presence of other prevailing yeast genera, such as Candida or Saccharomyces [23, 31].

\section{Conclusions}

Massive changes in gut bacteriome composition, explaining 20\% of total GB variation, have been documented in several previous studies following introduction of MM from commensal populations to breeding facilities [34]. These changes had a dramatic effect on host physiology, immune function and fitness [43, 61], with important implications on the usage of captive MM colonised by breeding facility-specific gastrointestinal microbiota as models for biomedical research. Similarly, the gut mycobiome profiles reported from captive MM appear to show pronounced differences to the commensal population GM described in this study [23, 31]. The fact that such massive changes in GB and associated host phenotype occurred between two human-associated lifestyles (commensal and captive) leads to the obvious assumption that transition from a wild-living to a commensal niche will have an even more dramatic effect. Nevertheless, our study comparing gut microbiota differences between commensal MM and non-commensal MS suggests relatively low differences in GB content and diversity between the two species, accounting for $<10 \%$ of total GB variation. Moreover, mouse GM was surprisingly uniform and consistently dominated by Kazachstania, both in commensal MM and non-commensal MS. Consequently, we suggest that translocation from a commensal population to captivity has a comparatively higher effect on mouse gut microbiota than gut microbiota changes associated with adaptation to a commensal niche. Further observation research (1) covering more commensal vs. non-commensal species pairs or (2) focusing on temporal dynamics of gut microbiota changes as well as (3) experimental studies aimed at direct microbiota manipulations would help to uncover details behind variation in gut microbiota vs. host physiology associated with commensal life style.

\section{Methods}

\section{Sample collection}

The mice used in this study were live-trapped in east Slovakia between the 7th and 9th of December 2015, with 4 individuals of MM trapped in a farm granary at
Drienovec ( $\mathrm{N} 48^{\circ} 36.683^{\prime}$, E $20^{\circ} 56.333^{\prime}$ ) and 8 individuals of MS obtained from field habitat near Drienovec

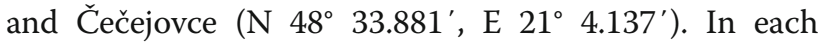
case, the traps were controlled twice per day, with trapped individuals taken back to the laboratory and caged separately in clean cages with sterile bedding to avoid microbial cross-infection. All mice were sacrificed by cervical dislocation and dissected within 12-h of trapping. Each of the three gut sections (ileum, caecum and colon) was placed on a sterile Petri dish. They were cut longitudinally. The content was gently washed out with sterile physiological saline solution and the tissue samples were placed separately into sterile cryotubes, rapidly frozen in liquid nitrogen and stored at $-80^{\circ} \mathrm{C}$. Whole compartments were taken from the caecum and colon, and the distal part only from the ileum $(1 \mathrm{~cm})$. Sampled species are not under legislative protection. Ethical statement regarding sample collection and experimental procedures is provided in "Declarations" section.

\section{Gut bacteriome genotyping}

Metagenomic DNA from gut tissue samples was extracted in April 2016 in a laminar flow cabinet using the PowerSoil DNA isolation kit (MO BIO Laboratories Inc., USA). We failed to collect a caecum sample from one MS individual; consequently, the final dataset included 35 samples of metagenomic DNA (Table S2).

Primers flanking the V3-V4 variable region on bacterial $16 \mathrm{~S}$ rRNA gene (i.e., S-D-Bact-0341-b-S-17 [CCTACGGGNGGCWGCAG] and S-D-Bact-0785-a-A21 [GACTACHVGGGTATCTAATCC]) were used during the polymerase chain reaction (PCR) step [62], both forward and reverse primers being tagged with 10bp barcodes for sample demultiplexing. For the PCR, we used $8.2 \mu \mathrm{l}$ of KAPA HIFI Hot Start Ready Mix (Kapa Biosystems, USA), $0.56 \mu \mathrm{M}$ of each primer and $6.2 \mu \mathrm{l}$ of DNA template. PCR conditions were as follows: initial denaturation at $98^{\circ} \mathrm{C}$ for $5 \mathrm{~min}$, followed by 30 cycles each of $98^{\circ} \mathrm{C}(15 \mathrm{~s}), 55^{\circ} \mathrm{C}(20 \mathrm{~s})$ and $72^{\circ} \mathrm{C}(40 \mathrm{~s})$ and a final extension at $72{ }^{\circ} \mathrm{C}(5 \mathrm{~min})$. The PCR products, together with negative controls (PCR products for blank DNA isolates), were run on $1.5 \%$ agarose gel, the concentration of the PCR product being assessed based on gel band intensity using GenoSoft software (VWR International, Belgium). The samples were subsequently pooled at equimolar concentration and run on 1.5\% agarose gel, with bands of appropriate size excised from the gel and purified using the High Pure PCR product Purification Kit (Roche, Switzerland), according to the manufacturer's instructions. Sequencing adaptors were ligated using TruSeq nano DNA library preparation kits (Illumina, USA) and the resulting amplicon libraries sequenced on a single MiSeq run (Illumina, USA) using v3 chemistry and $2 \times 300$ bp paired-end reads at Centre de 
Biologie pour la Gestion des Populations (CBGP, Montferrier sur Lez Cedex, France). Technical PCR duplicates were sequenced for individual samples.

\section{Gut mycobiome genotyping}

The ITS2 region was amplified using universal ITS3 (GCATCGATGAAGAACGCAGC) and ITS4 (TCCTCC GCTTATTGATATGC) primers [63] flanked by oligonucleotides compatible with Nextera adaptors (Illumina, USA). For the PCR reaction, we used $5 \mu \mathrm{l}$ KAPA HIFI Hot Start Ready Mix (Kapa Biosystems, USA), $0.2 \mu \mathrm{M}$ of each primer and $4.6 \mu \mathrm{l}$ of DNA template. PCR conditions were as follows: initial denaturation at $95^{\circ} \mathrm{C}$ for 3 min, followed by 30 cycles each of $95^{\circ} \mathrm{C}(30 \mathrm{~s}), 53^{\circ} \mathrm{C}(30$ s) and $72{ }^{\circ} \mathrm{C}(30 \mathrm{~s})$ and a final extension at $72{ }^{\circ} \mathrm{C}(5 \mathrm{~min})$. Nextera sequencing adaptors were appended to the resulting PCR products during the second PCR round using $10 \mu \mathrm{l}$ of KAPA HIFI Hot Start Ready Mix (Kapa Biosystems, USA), $2 \mu \mathrm{M}$ of each primer and $6 \mu \mathrm{l}$ of PCR product from the first PCR. PCR conditions were as follows: initial denaturation at $95^{\circ} \mathrm{C}$ for $3 \mathrm{~min}$, followed by 15 cycles each of $95^{\circ} \mathrm{C}(30 \mathrm{~s}), 55^{\circ} \mathrm{C}(30 \mathrm{~s})$ and $72{ }^{\circ} \mathrm{C}(30$ s) and a final extension at $72{ }^{\circ} \mathrm{C}(5 \mathrm{~min})$. PCR products of the second PCR were run on $1.5 \%$ agarose gel and pooled at equimolar concentration. The final library was cleaned up using Agencourt AmpureXP beads (Beckman Coulter Life Sciences). Products of the desired size (250-700 bp) were extracted by PipinPrep (Sage Science Inc., USA) and sequenced on Illumina Miseq (v3 kit, $300 \mathrm{bp}$ paired-end reads) at the Central European Institute of Technology (CEITEC), Masaryk University, Brno (Czech Republic). Technical PCR duplicates were sequenced for individual samples as in the GB analysis. We failed to amplify and sequence ITS2 in two DNA samples.

\section{Bioinformatics analysis}

Skewer [64] was used for both sample demultiplexing and detection and trimming of gene-specific primers. In the next step, reads of low quality (expected error rate per paired-end read $>1$ ) were eliminated. Dada2 [65] was used for denoising of quality-filtered reads and quantification of 16S rRNA and ITS2 haplotypes (hereafter operational taxonomic units; OTUs) in each sample. Next, UCHIME [66] was employed for detection of chimeric OTUs. The gold.fna (available at: https:// drive5.com/uchime/gold.fa) and UNITE databases [67] were used as references for chimeras filtering from the GB and GM datasets, respectively. After elimination of chimeric haplotypes, taxonomy for non-chimeric OTUs was assigned at $80 \%$ posterior confidence by RDP Classifier [68]. The GreenGenes database version gg.13.8 [69] was used for bacterial OTU annotation, and the UNITE database [67] for fungal OTU annotation. Bacterial OTU sequences were aligned using PyNast [70] and their phylogenetic tree constructed using FastTree [71]. We did not conduct phylogenetic reconstruction for ITS2 OTUs as ITS2 evolution is driven to a large extent by insertions and deletions, which complicates identification of homologous positions for phylogenetically disparate taxa. OTU tables (i.e. OTU read counts in individual samples), OTU sequences, their taxonomic annotations and phylogeny along with sample metadata were merged into separate GB and GM databases using the package phyloseq [72] in R (R Core Team 2015).

\section{Statistical analysis}

The GM database comprised 589,406 high-quality sequences grouped in 153 non-chimeric OTUs. The number of ITS2 reads per sample ranged between 1826 and 32,002 (median $=17,896$ ). Only basic descriptive tools were used in GM analysis as the sample structure was highly homogenous (detailed below). The GB database comprised 1,088,282 high quality sequences grouped in 1634 non-chimeric OTUs. As the number of bacterial reads varied between samples (range $=1415-58,106$, median $=29,188$ ), we rarefied the OTU table to achieve even sequencing depth per sample and used the downsampled database for further statistical analysis unless otherwise stated. The Shannon index was used as an alpha diversity measure, with per-sample Shannon diversities included as a response variable in generalised linear mixed effect models (GLMM), while sex, sampling location, gut section and species identity were included as alpha diversity predictors. We also tested for potential effects of species vs. gut section interaction. Individual identity was included a random term in order to account for pseudo-replication as multiple gut sections were analysed for each individual. Nonsignificant predictors were eliminated from the initial model in a step-wise manner in order to obtain the minimal adequate model [73]. Marginal effects for significant predictors (i.e. effect of predictor in questions controlled for the effects of other significant predictors) are reported. Dissimilarity based on OTU prevalence (binary Jaccard index) and relative abundance (Bray-Curtis index) was used to study divergence in microbiota composition between samples. First, we visualised the between-sample divergence pattern using Principal Coordinate Analysis (PCoA), then applied PERMANOVA (adonis2 function from the vegan $\mathrm{R}$ package) to test whether predictors already included in the alpha diversity analysis drove variation in microbial profile composition. To account for within-individual covariance, individual identity was included as a constraint for permutations (i.e. strata). We reported marginal probability values, i.e. significance of the variable in question controlled for the effect of all other variables included in the PERMANOVA model. To identify OTUs 
exhibiting variation in abundance between different gut sections and between the two species, we employed mixed models (from R package BhGLM [74]) assuming negative binomial error distribution. These analyses were only conducted for a subset of dominant OTUs (represented by $>0.1 \%$ reads and present in $>5$ samples, $n=$ 119). The marginal effect of the predictor in question (i.e. effect of species identity statistically controlled for systematic variation between gut sections and vice versa) was tested using likelihood-ratio tests, with qvalue [75] used as a multiple testing correction method. As in the previous analyses, individual identity was included as a random effect. Tukey post-hoc tests were employed after likelihood ratio testing to identify specific gut section pairs where the OTU in question exhibited significant variation in abundance. In specific cases, phylogenetic placement was used to provide a more detailed insight into OTU taxonomy. A set of reference $16 \mathrm{~S}$ rRNA sequences exhibiting $>97 \%$ similarity with the OTUs in question was extracted from the Silva database v. 132 [76]. Sequences were aligned using mafft [77] and a phylogenetic tree constructed using RAxML [78]. Bootstrap analysis based on 1000 replications was used to estimate support for the tree nodes.

\section{Supplementary information}

Supplementary information accompanies this paper at https://doi.org/10. 1186/s12866-020-01859-8.

Additional file 1: Figure S1. Phylogenetic placement of two Helicobacter operational taxonomic units whose abundances varied between Mus musculus (MM) and Mus spicilegus (MS). The tree of partial $16 \mathrm{~S}$ rRNA sequences was constructed using maximum likelihood. Bootstrap values are based on 1000 replicates. The scale bar represents a 0.04 (4\%) nucleotide sequence difference.

Additional file 2: Figure S2. Composition of the murine gut mycobiome. Proportion of dominant classes in mouse-mycobiome samples and bird-mycobiome samples (ten barn swallows [Hirundo rustica]; see Kreisinger et al. 2017 [40] for details) sequenced in parallel with the mouse samples.

Additional file 3: Table S1. Average proportions of bacterial taxa detected in three gut sections (ileum, caecum and colon) of two wild mouse species (Mus musculus, Mus spicilegus).

Additional file 4: Table S2. Sample metadata and sequencing data accession numbers.

\section{Abbreviations}

MM: Mus musculus; MS: Mus spicilegus; GB: Gut bacteriome; GM: Gut mycobiome; LME: Linear mixed effect models; PCoA: Principal Coordinate Analysis; OTU: Operational taxonomic unit; PCR: Polymerase chain reaction; CEITEC: Central European Institute of Technology

\section{Acknowledgements}

Access to computing and storage facilities owned by parties and projects contributing to the National Grid Infrastructure MetaCentrum, provided under the programme "Projects of Large Infrastructure for Research, Development, and Innovations" (LM2010005), is greatly appreciated. We acknowledge the Core Facility Genomics, Central European Institute of Technology, Masaryk University, supported by the National Center for Medical Genomics research infrastructure (LM2015091 funded by Ministerstvo Školství, Mládeže a Tělovýchovy, Czech Republic), and especially
Filip Pardy and Boris Tichý for their support while obtaining the scientific data presented in this paper.

\section{Authors' contributions}

JK and JP designed the study. LD carried out the field sampling. BB, LS and JFM performed the laboratory analysis. JK, BB and LS performed the data analysis. JK and JP secured funding. JK, BB and DC drafted the manuscript. All authors provided helpful comments and recommendations and approved the final version of the manuscript.

\section{Funding}

This study was supported through the Czech Science Foundation, Project no. 18-17796Y, and the Grant Agency of Charles University, Project no. 1501218. In addition, BB and LS were supported by SW Project no. 260571/2020.

\section{Availability of data and materials}

Sequencing data associated with this project are archived in European Nucleotide Archive (project accession numbers: PRJEB37682 and

PRJEB37678). Accession numbers for each sample are available in Table S2.

\section{Ethics approval and consent to participate}

Care of all mice and all procedures and experiments were performed according to National Institute of Health and the Office of Laboratory Animal Welfare guidelines for appropriate animal husbandry (OLAW assurance A3292-01), with all handling of live mice undertaken by an authorised person.

Consent for publication

Not applicable.

\section{Competing interests}

The authors declare that they have no competing interests.

\section{Author details}

${ }^{1}$ Department of Zoology, Faculty of Science, Charles University, Prague, Czech Republic. 'Studenec Research Facility, Institute of Vertebrate Biology, Czech Academy of Sciences, Brno, Czech Republic. ${ }^{3}$ CBGP, Montpellier SupAgro, INRA, CIRAD, IRD, Univ Montpellier, Montferrier-sur-Lez, France.

Received: 3 December 2019 Accepted: 16 June 2020

Published online: 06 July 2020

\section{References}

1. Cucchi T, Auffray J-C, Vigne J-D. On the origin of the house mouse synanthropy and dispersal in the near east and Europe: zooarcheological review and perspectives. In: Macholan M, Baird SJE, Munclinger P, Pialek J, editors. Evolution of the House Mouse. Cambridge: Cambridge University Press; 2012. p. 65-93.

2. Driscoll CA, Macdonald DW, O'Brien SJ. From wild animals to domestic pets, an evolutionary view of domestication. Proc Natl Acad Sci. 2009;106:9971-8. https://doi.org/10.1073/pnas.0901586106.

3. Hu Y, Hu S, Wang W, et al. Earliest evidence for commensal processes of cat domestication. Proc Natl Acad Sci. 2014;111:116-20. https://doi.org/10.1073/ pnas.1311439110.

4. Shapira I, Sultan H, Shanas U. Agricultural farming alters predator-prey interactions in nearby natural habitats. Anim Conserv. 2008;11:1-8. https:// doi.org/10.1111/j.1469-1795.2007.00145.x.

5. Morgan M. Methicillin-resistant Staphylococcus aureus and animals: zoonosis or humanosis? J Antimicrob Chemother. 2008;62:1181-7. https:// doi.org/10.1093/jac/dkn405.

6. Hendry AP, Farrugia TJ, Kinnison MT. Human influences on rates of phenotypic change in wild animal populations. Mol Ecol. 2008;17:20-9. https://doi.org/10.1111/j.1365-294X.2007.03428.x

7. Hemmer $\mathrm{H}$. Domestication: the decline of environmental appreciation. Cambridge: Cambridge University Press; 1990.

8. O'Connor TP. Working at relationships: another look at animal domestication. Antiquity. 1997;71:149-56. https://doi.org/10.1017/ S0003598X00084635.

9. Leach HM. Human domestication reconsidered. Curr Anthropol. 2003:44 349-68. https://doi.org/10.1086/368119. 
10. Dobney K, Larson G. Genetics and animal domestication: new windows on an elusive process. J Zool. 2006;269:060222013030001-???. https://doi.org/ 10.1111/j.1469-7998.2006.00042.x.

11. Varudkar A, Ramakrishnan U. Gut microflora may facilitate adaptation to anthropic habitat: a comparative study in Rattus. Ecol Evol. 2018;8:6463-72. https://doi.org/10.1002/ece3.4040.

12. Sekirov I, Russell SL, Antunes LCM, Finlay BB. Gut microbiota in health and disease. Physiol Rev. 2010;90:859-904. https://doi.org/10.1152/physrev.00045. 2009

13. Wallace TC, Guarner F, Madsen K, et al. Human gut microbiota and its relationship to health and disease. Nutr Rev. 2011;69:392-403. https://doi. org/10.1111/j.1753-4887.2011.00402.x.

14. Cho I, Blaser MJ. The human microbiome: at the interface of health and disease. Nat Rev Genet. 2012;13:260-70. https://doi.org/10.1038/nrg3182

15. Turnbaugh PJ, Ley RE, Mahowald MA, et al. An obesity-associated gut microbiome with increased capacity for energy harvest. Nature. 2006;444: 1027-31. https://doi.org/10.1038/nature05414

16. Cryan JF, Dinan TG. Mind-altering microorganisms: the impact of the gut microbiota on brain and behaviour. Nat Rev Neurosci. 2012;13:701-12 https://doi.org/10.1038/nrn3346.

17. Häcker $\mathrm{G}$, Redecke $\mathrm{V}$, Häcker H. Activation of the immune system by bacterial CpG-DNA. Immunology. 2002;105:245-51. https://doi.org/10.1046/j. 0019-2805.2001.01350.x.

18. Tlaskalová-Hogenová H, Štěpánková R, Hudcovic T, et al. Commensal bacteria (normal microflora), mucosal immunity and chronic inflammatory and autoimmune diseases. Immunol Lett. 2004;93:97-108. https://doi.org/10. 1016/j.imlet.2004.02.005.

19. Round JL. Shaping up with the gut microbiota. Sci Transl Med. 2014;6: 263ec201. https://doi.org/10.1126/scitranslmed.aaa2064.

20. Taylor KD, Strom SP, Funari VA, et al. Interactions between commensal Fungi and the C-type Lectin receptor Dectin-1 influence colitis. Science. 2012;336:1314-7. https://doi.org/10.1126/science.1221789.

21. Turnbaugh PJ, Ridaura VK, Faith JJ, et al. The effect of diet on the human gut microbiome: a metagenomic analysis in humanized Gnotobiotic mice. Sci Transl Med. 2009;1:6ra14. https://doi.org/10.1126/ scitransImed.3000322.

22. Walk ST, Blum AM, Ewing SA-S, et al. Alteration of the murine gut microbiota during infection with the parasitic helminth Heligmosomoides polygyrus. Inflamm Bowel Dis. 2010;16:1841-9. https://doi.org/10.1002/ibd. 21299.

23. Heisel T, Montassier E, Johnson A, et al. High-fat diet changes fungal microbiomes and Interkingdom relationships in the murine gut. mSphere. 2017;2:1-14. https://doi.org/10.1128/mSphere.00351-17.

24. Dhanasiri AKS, Brunvold L, Brinchmann MF, et al. Changes in the intestinal microbiota of wild Atlantic cod Gadus morhua L. upon captive rearing. Microb Ecol. 2011;61:20-30. https://doi.org/10.1007/s00248-010-9673-y.

25. Kohl KD, Dearing MD. Wild-caught rodents retain a majority of their natural gut microbiota upon entrance into captivity. Environ Microbiol Rep. 2014;6: 191-5. https://doi.org/10.1111/1758-2229.12118.

26. Metcalf JL, Song SJ, Morton JT, et al. Evaluating the impact of domestication and captivity on the horse gut microbiome. Sci Rep. 2017;7:1-9. https://doi. org/10.1038/s41598-017-15375-9.

27. McKenzie VJ, Kowalewski M, Di Fiore A, et al. The effects of captivity on the mammalian gut microbiome. Integr Comp Biol. 2017;57:690-704. https://doi. org/10.1093/icb/icx090.

28. Aplin KP, Suzuki H, Chinen AA, et al. Multiple geographic origins of commensalism and complex dispersal history of black rats. PLoS One. 2011; 6:e26357. https://doi.org/10.1371/journal.pone.0026357.

29. Ohnishi N, Yuasa H, Tanaka S, et al. Transgenic expression of Helicobacter pylori CagA induces gastrointestinal and hematopoietic neoplasms in mouse. Proc Natl Acad Sci. 2008;105:1003-8. https://doi.org/10.1073/pnas. 0711183105

30. Kau AL, Ahern PP, Griffin NW, et al. Human nutrition, the gut microbiome and the immune system. Nature. 2011;474:327-36. https://doi.org/10.1038/ nature10213.

31. Iliev ID, Funari VA, Taylor KD, et al. Interactions between commensal Fungi and the C-type Lectin receptor Dectin-1 influence colitis. Science. 2012;336: 1314-7. https://doi.org/10.1126/science.1221789.

32. Gu S, Chen D, Zhang J-NN, et al. Bacterial community mapping of the mouse gastrointestinal tract. PLoS One. 2013;8:e74957. https://doi.org/10. 1371/journal.pone.0074957.
33. Linnenbrink M, Wang J, Hardouin EA, et al. The role of biogeography in shaping diversity of the intestinal microbiota in house mice. Mol Ecol. 2013; 22:1904-16. https://doi.org/10.1111/mec.12206.

34. Kreisinger J, Čížková D, Vohánka J, Piálek J. Gastrointestinal microbiota of wild and inbred individuals of two house mouse subspecies assessed using high-throughput parallel pyrosequencing. Mol Ecol. 2014;23:5048-60. https://doi.org/10.1111/mec.12909.

35. Suzuki TA, Nachman MW. Spatial heterogeneity of gut microbial composition along the gastrointestinal tract in natural populations of house mice. PLoS One. 2016;11:1-15. https://doi.org/10.1371/journal.pone.0163720.

36. Kreisinger J, Bastien G, Hauffe $\mathrm{HC}$, et al. Interactions between multiple helminths and the gut microbiota in wild rodents. Philos Trans R Soc B Biol Sci. 2015;370:20140295. https://doi.org/10.1098/rstb.2014.0295.

37. Siriyappagouder P, Kiron V, Lokesh J, et al. The intestinal Mycobiota in wild Zebrafish comprises mainly Dothideomycetes while Saccharomycetes predominate in their laboratory-reared counterparts. Front Microbiol. 2018;9: 1-13. https://doi.org/10.3389/fmicb.2018.00387.

38. Sarver B, Keeble S, Cosart T, Tucker PK, Dean MD, Good JM. Phylogenomic insights into mouse evolution using a pseudoreference approach. Genome Biol Evol. 2017;9:726-39.. https://doi.org/10.1093/gbe/evx034.

39. Sokolov VE, Kotenkova EV, Michailenko AG. Mus spicilegus. Mamm Species. 1998;592:1-6. https://doi.org/10.2307/3504484.

40. Kreisinger J, Kropáčková L, Petrželková A, et al. Temporal stability and the effect of Transgenerational transfer on fecal microbiota structure in a long distance migratory bird. Front Microbiol. 2017;8:1-19. https://doi.org/10. 3389/fmicb.2017.00050.

41. Scupham AJ, Patton TG, Bent E, Bayles DO. Comparison of the Cecal microbiota of domestic and wild turkeys. Microb Ecol. 2008;56:322-31. https://doi.org/10.1007/s00248-007-9349-4.

42. Clayton JB, Vangay $P$, Huang $H$, et al. Captivity humanizes the primate microbiome. Proc Natl Acad Sci. 2016;113:10376-81. https://doi.org/10.1073/ pnas.1521835113.

43. Rosshart SP, Vassallo BG, Angeletti D, et al. Wild mouse gut microbiota promotes host fitness and improves disease resistance. Cell. 2017;171:10151028.e13. https://doi.org/10.1016/j.cell.2017.09.016.

44. Kohl KD, Skopec MM, Dearing MD. Captivity results in disparate loss of gut microbial diversity in closely related hosts. Conserv Physiol. 2014;2:1-11. https://doi.org/10.1093/conphys/cou009.

45. Flint HJ, Scott KP, Louis P, Duncan SH. The role of the gut microbiota in nutrition and health. Nat Rev Gastroenterol Hepatol. 2012;9:577-89. https:// doi.org/10.1038/nrgastro.2012.156.

46. Lu H-P, Lai Y-C, Huang S-W, et al. Spatial heterogeneity of gut microbiota reveals multiple bacterial communities with distinct characteristics. Sci Rep. 2015;4:6185. https://doi.org/10.1038/srep06185.

47. Mackie Rl, Aminov Rl, Hu W, et al. Ecology of uncultivated Oscillospira species in the rumen of cattle, sheep, and reindeer as assessed by microscopy and molecular approaches. Appl Environ Microbiol. 2003;69: 6808-15. https://doi.org/10.1128/AEM.69.11.6808-6815.2003.

48. Konikoff T, Gophna U. Oscillospira: a central, enigmatic component of the human gut microbiota. Trends Microbiol. 2016;24:523-4. https://doi.org/10. 1016/j.tim.2016.02.015.

49. Gophna U, Konikoff T, Nielsen HB. Oscillospira and related bacteria-from metagenomic species to metabolic features. Environ Microbiol. 2017;19:83541. https://doi.org/10.1111/1462-2920.13658.

50. Gomez-Arango LF, Barrett HL, McIntyre HD, et al. Increased systolic and diastolic blood pressure is associated with altered gut microbiota composition and butyrate production in early pregnancy. Hypertension. 2016;68:974-81. https://doi.org/10.1161/HYPERTENSIONAHA.116.07910.

51. Noble EE, Hsu TM, Kanoski SE. Gut to brain Dysbiosis: mechanisms linking Western diet consumption, the microbiome, and cognitive impairment. Front Behav Neurosci. 2017;11:1-10. https://doi.org/10.3389/fnbeh.2017. 00009.

52. Wasimuddin ČD, Bryja J, et al. High prevalence and species diversity of helicobacter spp. detected in wild house mice. Appl Environ Microbiol. 2012;78:8158-60. https://doi.org/10.1128/AEM.01989-12.

53. Ding S-ZSZ, Minohara Y, Xue JF, et al. Helicobacter pylori infection induces oxidative stress and programmed cell death in human gastric epithelial cells. Infect Immun. 2007;75:4030-9. https://doi.org/10.1128/IAl.00172-07.

54. Linz B, Balloux F, Moodley $Y$, et al. An African origin for the intimate association between humans and helicobacter pylori. Nature. 2007:445:9158. https://doi.org/10.1038/nature05562.An. 
55. Atherton JC, Blaser MJ. Coadaptation of helicobacter pylori and humans: ancient history, modern implications. J Clin Invest. 2009;119:2475-87. https://doi.org/10.1172/JCl38605.

56. Kurtzman CP, Robnett CJ, Ward JM, et al. Multigene phylogenetic analysis of pathogenic Candida species in the Kazachstania (Arxiozyma) telluris complex and description of their ascosporic states as Kazachstania bovina sp. nov., K. heterogenica sp. nov., K. pintolopesii sp. nov., and K. slooffiae. J Clin Microbiol. 2005;43:101-11. https://doi.org/10.1128/JCM.43.1.101-111. 2005.

57. Urubschurov V, Janczyk P, Souffrant W-B, et al. Establishment of intestinal microbiota with focus on yeasts of unweaned and weaned piglets kept under different farm conditions. FEMS Microbiol Ecol. 2011;77:493-502. https://doi.org/10.1111/j.1574-6941.2011.01129.x.

58. Urubschurov $V$, Büsing $K$, Freyer $G$, et al. New insights into the role of the porcine intestinal yeast, Kazachstania slooffiae, in intestinal environment of weaned piglets. FEMS Microbiol Ecol. 2017;93:1-12. https://doi.org/10.1093/ femsec/fiw245.

59. Flahou B, De Baere T, Chiers K, et al. Gastric infection with kazachstania heterogenica influences the outcome of a helicobacter suis infection in mongolian gerbils. Helicobacter. 2010;15:67-75. https://doi.org/10.1111/j. 1523-5378.2009.00736x

60. Alvarez-Perez $S$, Mateos A, Dominguez $L$, et al. First isolation of the anamorph of Kazachstania heterogenica from a fatal infection in a primate host. Med Mycol. 2012;50:193-6. https://doi.org/10.3109/13693786.2011. 578155.

61. Beura LK, Hamilton SE, Bi K, Schenkel JM, Odumade OA, Casey CA, Thompson EA, Fraser KA, Rosato PC, Filali-Mouhim A, Sekaly RP, Jenkins MK, Vezys $V$, Haining WN, Jameson SC, Masopust D. Normalizing the environment recapitulates adult human immune traits in laboratory mice. Nature. 2016;532:512-6. https://doi.org/10.1038/nature17655.

62. Klindworth A, Pruesse $E$, Schweer $T$, et al. Evaluation of general $16 \mathrm{~S}$ ribosomal RNA gene PCR primers for classical and next-generation sequencing-based diversity studies. Nucleic Acids Res. 2013;41:e1. https:// doi.org/10.1093/nar/gks808.

63. White T, Bruns T, Lee S, Taylor J. Amplification and direct sequencing of fungal ribosomal RNA genes for phylogenetics. In: Innis MA, Gelfand DH, Sninsky JJ, White TJ, editors. PCR protocols: a guide to methods and applications. New York: Academic Press Inc; 1990. p. 315-22.

64. Jiang H, Lei R, Ding S-W, et al. Skewer: a fast and accurate adapter trimmer for next-generation sequencing pairedend reads. BMC Bioinformatics. 2014; 15:1-12. https://doi.org/10.1186/1471-2105-15-182.

65. Callahan BJ, McMurdie PJ, Rosen MJ, et al. DADA2: high-resolution sample inference from Illumina amplicon data. Nat Methods. 2016;13:581-3. https:// doi.org/10.1038/nmeth.3869.

66. Edgar RC, Haas BJ, Clemente JC, et al. UCHIME improves sensitivity and speed of chimera detection. Bioinformatics. 2011;27:2194-200. https://doi. org/10.1093/bioinformatics/btr381.

67. Kõljalg U, Nilsson RH, Abarenkov K, et al. Towards a unified paradigm for sequence-based identification of fungi. Mol Ecol. 2013;22:5271-7. https:// doi.org/10.1111/mec.12481.

68. Wang Q, Garrity GM, Tiedje JM, Cole JR. Naıve Bayesian classifier for rapid assignment of rRNA sequences. Appl Environ Microbiol. 2007;73:5261-7. https://doi.org/10.1128/AEM.00062-07.

69. DeSantis TZ, Hugenholtz P, Larsen N, et al. Greengenes, a chimera-checked 165 rRNA gene database and workbench compatible with ARB. Appl Environ Microbiol. 2006;72:5069-72. https://doi.org/10.1128/AEM.03006-05.

70. Caporaso JG, Bittinger K, Bushman FD, et al. PyNAST: a flexible tool for aligning sequences to a template alignment. Bioinformatics. 2010;26:266-7. https://doi.org/10.1093/bioinformatics/btp636

71. Price MN, Dehal PS, Arkin AP. FastTree: computing large minimum evolution trees with profiles instead of a distance matrix. Mol Biol Evol. 2009;26:164150. https://doi.org/10.1093/molbev/msp077.

72. McMurdie PJ, Holmes S. Phyloseq: an R package for reproducible interactive analysis and graphics of microbiome census data. PLoS One. 2013;8:e61217. https://doi.org/10.1371/journal.pone.0061217.

73. Crawley MJ. The R book. New York: Wiley Publishing; 2007.

74. Yi N, Liu N, Zhi D, Li J. Hierarchical generalized linear models for multiple groups of rare and common variants: jointly estimating group and individual-variant effects. PLoS Genet. 2011;7:e1002382. https://doi.org/10. 1371/journal.pgen.1002382.
75. Storey JD, Tibshirani R. Statistical significance for genomewide studies. Proc Natl Acad Sci. 2003;100:9440-5. https://doi.org/10.1073/pnas.1530509100.

76. Quast C, Pruesse E, Yilmaz P, et al. The SILVA ribosomal RNA gene database project: improved data processing and web-based tools. Nucleic Acids Res. 2013;41:590-6. https://doi.org/10.1093/nar/gks1219.

77. Katoh K, Standley DM. MAFFT multiple sequence alignment software version 7: improvements in performance and usability. Mol Biol Evol. 2013; 30:772-80. https://doi.org/10.1093/molbev/mst010.

78. Stamatakis A. RAxML version 8: a tool for phylogenetic analysis and postanalysis of large phylogenies. Bioinformatics. 2014;30:1312-3. https://doi.org/ 10.1093/bioinformatics/btu033.

\section{Publisher's Note}

Springer Nature remains neutral with regard to jurisdictional claims in published maps and institutional affiliations.
Ready to submit your research? Choose BMC and benefit from:

- fast, convenient online submission

- thorough peer review by experienced researchers in your field

- rapid publication on acceptance

- support for research data, including large and complex data types

- gold Open Access which fosters wider collaboration and increased citations

- maximum visibility for your research: over $100 \mathrm{M}$ website views per year

At $\mathrm{BMC}$, research is always in progress.

Learn more biomedcentral.com/submissions 